Z. klin. Chem. u. klin. Biochem.

8. Jg., S. 124-126, März 1970

\title{
Isoenzyme der Fructose-Phosphat-Aldolase bei Kranken mit Tumoren im Uro-Genital-System
}

\author{
Isoenzyme der Fructose-Phosphat-Aldolase. Mitteilung XII ) \\ Von A. L. Dikow und L. Donowskr \\ Aus der Biocbemischen Abteilung des Onkologischen Forschungsinstituts (Direktor: Prof. N. Antscbew) \\ und dem Lebrstubl für Urologie der Medizinischen Hocbscbule (Leiter: Prof. S. Lambrew), Sofia, Bulgarien
}

(Eingegangen am 25. September 1969)

\begin{abstract}
In vorliegender Arbeit wird die Gesamtaktivität und das Isoenzymmuster der Aldolase in dèn Tumoren und im Serum von Kranken mit verschiedenen Karzinomen im Urogenitalsystem untersucht. Am höchsten ist die Aldolaseaktivität im Papillom und Karzinom der Harnblase und wesentlich niedriger in den Nieren- und Hodenkarzinomen. Die Serumaldolase ist am höchsten bei den Patienten mit Harnblasenkarzinom und am niedrigsten, wenn auch noch über der Norm, bei den Patienten mit Papillom der Harnblase. Das Issoenzymmuster der Aldolase in den Tumoren besteht aus intensiven Fraktionen vom Typ Muskel „A“ Aldolase, die nach ihrer elektrophoretischen Bewreglichkeit den im Serum von Kranken hervortretenden Fraktionen entsprechen. Auf Grund der erhaltenen Ergebnisse und der Literaturangaben werden Schlüsse über die aus den Tumoren herkommende erhöhte Serumaldolase bei Kranken mit Karzinomen im Urogenitalsystem gezogen.
\end{abstract}

\section{Isoenzymes of fructose phosphate aldolase in patients with tumours of the urogenital system}

The total activity and the isoenzyme pattern of aldolase in the tumours and in the serum of patients with various carcinomas of the urogenital system were studied. The highest aldolase activities were found in papilloma and carcinoma of the bladder and essentially lower values in carcinomas of the kidneys and gonads. Serum aldolase is highest in patients with bladder carcinoma and lowest, though still higher than normal, in patients with papilloma of the bladder. The isoenzyme pattern of the aldolase from the tumours shows intense fractions of muscle type "A" aldolase, which, according to their electrophoretic migration, correspond to the fractions that appear in the sera of the patients. On the basis of these results and data in the literature, the elevation of serum aldolase by the production of aldolase in the tumours of patients with carcinoma of the urogenital system is discussed.

Die Bestimmung der Serumaldolase bei Kranken mit Tumoren im Urogenital-Trakt nimmt einen wichtigen Platz in der Enzymdiagnostik des Krebses ein. Verschiedene Verfasser konnten eine erhöhte Aldolase. an Kranken mit Karzinomen der Harnblase $(1,2)$, der Prostata $(2,3,4,5,6,7)$ und des Hodens (8) nachweisen. In unseren früheren Veröffentlichungen untersuchten wir das Isoenzymmuster der Serumaldolase an Gesunden (9) sowie von Kranken mit progressiver Muskeldystrophie (10), mit infektiöser Hepatitis (11) und verschiedenen bösartigen Hämopathien (12). Dabei konnten, der erhöhten Aldolase entsprechend, auch die Veränderungen im Isoenzymmuster im Vergleich zu den Normalwerten nachgewiesen werden.

Im Rahmen dieser Forschungen setzten wir uns mit vorliegender Arbeit zum Ziel, die Gesamtaktivität und das Isoenzymmuster der Aldolase im Serum und in den Tumoren bei Kranken mit Karzinomen und Papillomen der Harnblase sowie mit Nieren- und Hodenkarzinomen $\mathrm{zu}$ untersuchen.

\section{Material und Methoden}

Wir untersuchten 33 unbehandelte Kranke mit primären, nicht metastasierten Tumoren im Urogenital-System, darunter 10 Harnblasen-Karzinome, 8 Harnblasen-Papillome, 10 NierenKarzinome und 5 Hoden-Karzinome. Den Patienten wurde zweimal Blut entnommen - vor der operativen Beseitigung des

1) Mitteilung XI, siehe Drkow, A. L. u. A. Lasarowa, diese Z. $8,122(1970)$.
Tumors und 14 Tage später. Im Serum wurde die Gesamt-Aldolaseaktivität mit dem "Aldolase-UV-Test" der Fa. Boehringer, Mannheim, bestimmt. Von den operativ entfernten Tumoren nahmen wir Material, homogenisierten es bei $0^{\circ}$ und zentrifugierten $30 \mathrm{Min}$. bei $150000 \mathrm{~g}$ und $0^{\circ}$. Im klaren Uberstand wurde das Gesamteiweiß und die Aldolaseaktivität gegenüber Fructose1,6-diphosphat (FDP) nach früher beschriebenem Verfahren bestimmt (13). Die elektrophoretische Trennung der Isoenzyme der Aldolase im Homogenat der Tumoren und im Serum erfolgte auf $0,6 \%$ Agarosegel mit Tris/EDTA/Borsäure-Puffer $\mathrm{pH} 8,9$ (13), die Darstellung der Isoenzymfraktionen nach früher von Dikow vorgeschlagener Methode (9).

\section{Ergebnisse und Diskussion}

Die Bestimmungsergebnisse der Gesamtaldolaseaktivität bei Kranken mit Tumoren im Urogenitalsystem sind auf Tabelle 1 dargestellt.

Tab. 1

Gesamtaktivität der Fructose-Phosphat-Aldolase in Tumoren des Urogenitalsystems (A) in Mikromol FDP/mg GewebseiweiB pro Min bei $37^{\circ}$ und im Serum von Kranken vor (B) und nach der Operation (C) in $\mathrm{mU} / \mathrm{m}$

\begin{tabular}{lcccc}
\hline \multicolumn{1}{c}{ Tumorart } & Anzahl & A & B & C \\
\hline Carcinoma vesicae urinariae & 10 & $\mathbf{0 , 8 0}$ & $\mathbf{8 , 8 1}$ & $\mathbf{4 , 8 2}$ \\
Papilloma vesicae urinariae & 8 & $\mathbf{0 0 9}$ & 6,17 & $\mathbf{4}, 63$ \\
Carcinoma renis & 10 & 0,61 & $\mathbf{7 , 2 4}$ & $\mathbf{4 , 5 4}$ \\
Carcinoma testis & 5 & 0,55 & $\mathbf{6 , 4 7}$ & $\mathbf{3 , 9 7}$ \\
Gesunde & 50 & - & $\mathbf{3 , 5 0} \pm 0,63$ & - \\
\hline
\end{tabular}

Die Gesamtaldolaseaktivität in den untersuchten Tumoren ist am höchsten in den gutartigen, also im Papillom der Harnblase. Unter den bösartigen Tumoren ist die höchste Aldolaseaktivität im Karzinom der Harnblase zu beobachten und eine wesentlich verminderte in den 


\section{Standardmathoden}

itt
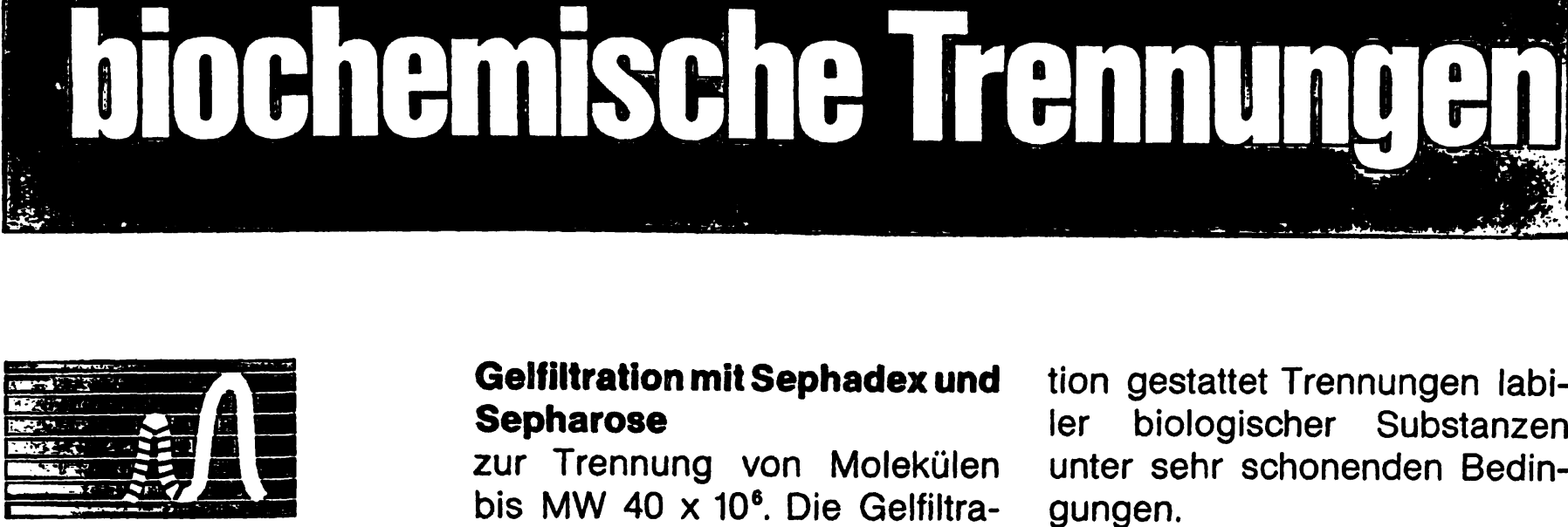

Gelfiltration mit Sephadex und Sepharose

zur Trennung von Molekülen bis MW $40 \times 10^{6}$. Die Gelfiltra- tion gestattet Trennungen labiler biologischer Substanzen unter sehr schonenden Bedingungen.

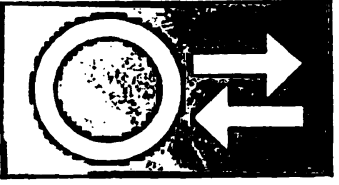

Ionenaustauscher-Chromatographie mit Sephadex-Ionenaustauschern,

die die Vorzüge von CelluloseundKunstharzaustauschern ver-

einigen. Charakteristisch sind hohe Kapazität, niedrige unspezifische Adsorption und ausgezeichnete Reproduzierbarkeit.

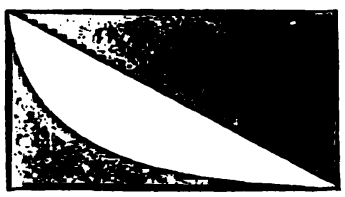

Vertellung in wäBrigen Pha-

zur Fraktionierung sehr hochsen-Systemen mit Dextran und Dextran-Derivaten

molekularer Stoffe wie Viren, Nukleinsäuren und Zellpartikeln unter sehr milden Bedingungen.

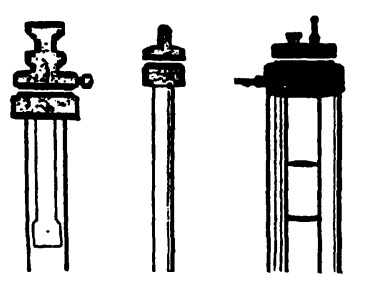

\section{Chromatographierohre.}

Unsere Chromatographierohre, die wir speziell für Gelfiltration und lonenaustausch-Chromato-

graphie entwickelt haben, ermöglichen reproduzierbare Trennresultate. Sie stehen Ihnen in großer Auswahl mit diversem Zubehör zur Verfügung.

\section{Literaturdienst}

Als Hilfe für Wissenschaftler geben wir einen umfassenden Literaturdienst heraus. Eine jährlich erscheinende Referenzliste enthält etwa 1000 neue
Literaturstellen. Bitte schreiben Sie uns, wenn wir Sie in unseren Verteiler aufnehmen sollen. Fordern Sie auch Broschüren über unsere Separationsprodukte und das Literaturverzeichnis an. 


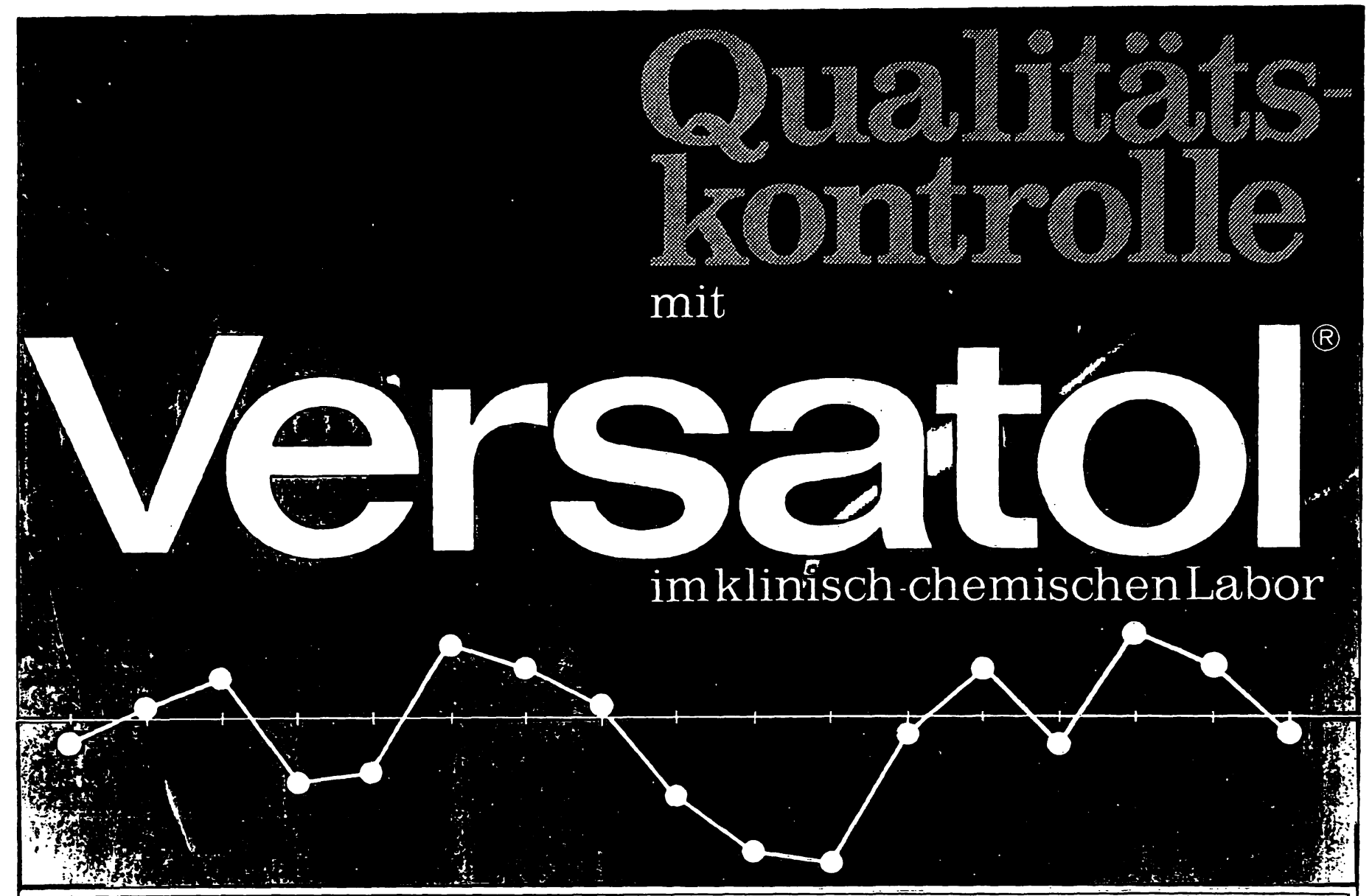

Uberwachung von Präzision und Richtigkeit im normalen und pathologischen Bereich

Bilirubin

Calcium

Chlorid

Freies Cholesterin

Gesamt-Cholesterin

Kreatinin

Glukose

Rest-N

Anorg. Phosphor

Eiweißgebundenes Jod

Kalium

Gesamt-Stickstoff

Gesamt-Eiweiß

Natrium

Harnstoff-N

Harnsäure

Alkalische Phosphatase

Saure Phosphatase

Amylase

Lipase

Transaminase GOT

Laktat-Dehydrogenase LDH

\section{GODECKE}




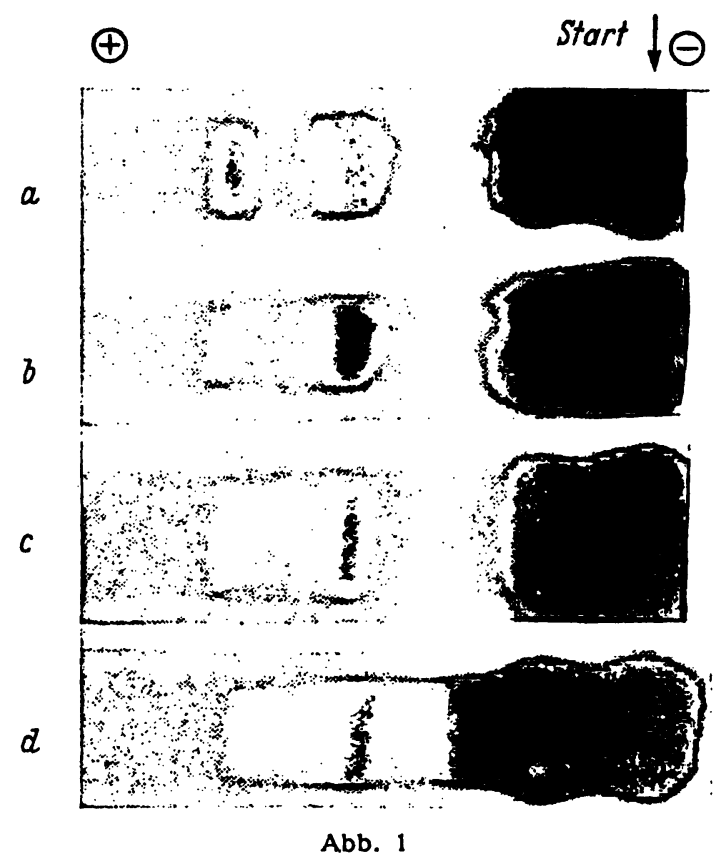

Isoenzymmuster der Fructose-Phosphat-Aldolase in Tumoren des Urogenitylsystems (Substrat Fructose-1,6-diphosphat) a) Harnblasen-Karzinom, b) Harnblasen-Papillom, c) Nieren-Karzi-

Nieren- und Hodenkarzinomen, wie auch von anderen Verfassern festgestellt wurde (14). Die Aldolasekativität im Serum der untersuchten Patienten übersteigt 2-3mal die Norm, wobei die höchsten Werte an Kranken mit Harnblasenkarzinom nachgewiesen wurden. Diese Resultate stimmen mit den Angaben anderer Autoren überein $(1,2,8)$. Bei den Patienten mit Papillom der Harnblase ist die Serumaldolase niedriger, wenn sie auch über dem Normalwert liegt.

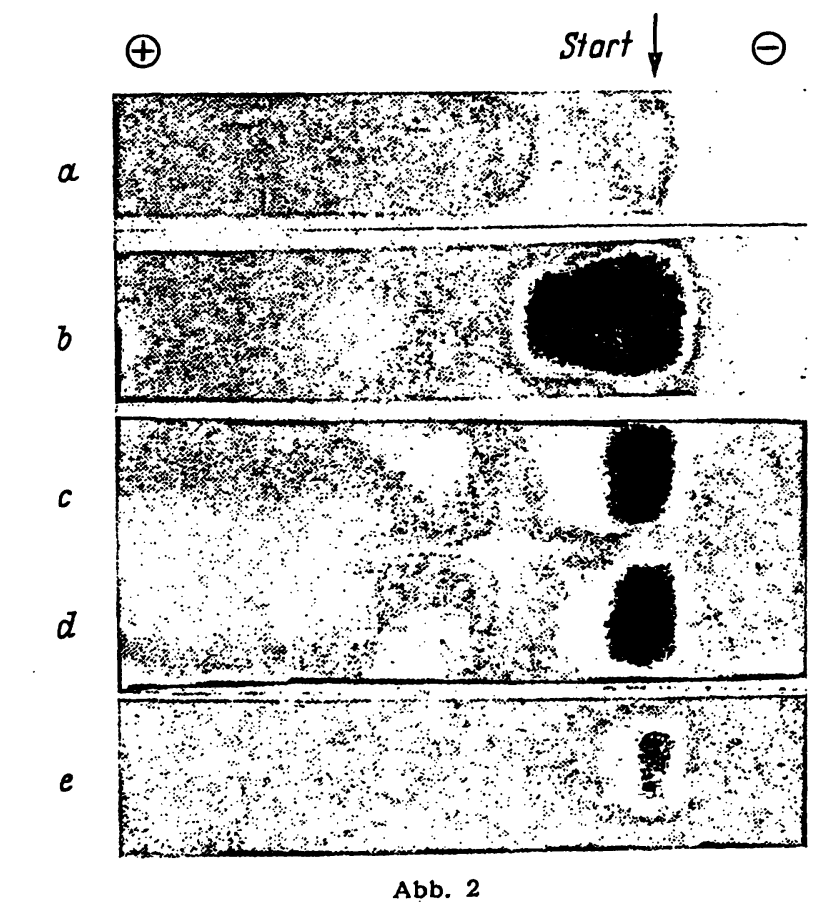

Isoenzymmuster der Fructose-Phosphat-Aldolase im Serum von Kranken mit Tumoren im Urogenitalsystem (Substrat Fructose-1,6diphosphat) a) bei Gesunden, b) bei Kranken mit Karzinom der Harnblase, c) bei der Niere, e) bei Kranken mit Testiskarzinom
Das Isoenzymmuster der Aldolase in den verschiedenen Tumoren des Urogenitalsystems ist auf Abbildung 1 veranschaulicht. Daraus ist zu ersehen, daß bei sämtlichen untersuchten Tumoren zwei breite und sehr intensive Fraktionen anodisch bei der Startlinien vom Typ Muskel „A“ Aldolase zu finden sind. Außerdem treten am anodischen Ende des Zymogramms ebenfalls je zwei Fraktionen hervor, die, von der Tumorart abhängig, nach Größe und Intensität variieren. Im Vergleich zum Isoenzymmuster der normalen Niere und Hoden (15) ist bemerkenswert, daß bei Tumoren dieser Organe die kathodisch liegende Isoenzymfraktion vom Typ Leber „B“ Aldolase fehlt. Wir konnten ebenfalls bei anderen Tumoren feststellen (16), daß ihr Isoenzymmuster vorwiegend aus anodisch bei der Startlinie liegenden Fraktionen vom Typ „A“ Aldolase besteht. Nur bei den Hirn- und Lebertumoren $(17,18)$ fanden wird deutlich dargestellte und intensive Fraktionen vom Typ Hirn "C" und Leber „B“ Aldolase.

Das Isoenzymmuster der Serumaldolase unserer Patienten ist aus Abbildung $2 \mathrm{zu}$ ersehen. Beim gesunden Menschen hat die Serumaldolase vier Isoenzymfraktionen. Nach ihrer elektrophoretischen Beweglichkeit entsprechen sie den Albuminen sowie den $\alpha_{2^{-}}, \beta_{1^{-}}$, und teilweise den $\gamma$-Globulinen im Serum, wobei die anodisch bei der Startlinie liegende Fraktion kaum bemerkbar ist und oft fehlt (Abb. 2a). Im Isoenzymmuster unserer Patienten wurden wesentliche Abweichungen von der Norm festgestellt. Die anodisch bei der Startlinie liegende Isoenzymfraktion erhöht mehrfach ihre Intensität und wiederum anodisch von ihr, treten $2-3$ neue ebenfalls intensive Fraktionen hervor (Abb. $2 b$, c, d, e). Diese Veränderungen sind besonders stark bei Kranken mit Harnblasenkarzinom. Die festgestellte Aktivitätserhöhung der Serumaldolase bei Kranken mit Tumoren im Urogenitaltrakt könnte mit dem Enzymaustritt in das Blut sowohl aus den schnellwachsenden und wegen mangelhafter Blutzufuhr leicht nekrotisierenden Tumorzellen, als auch aus den vom Tumorprozeß geschädigten regionalen Gewebe, was nach BoDANSKI (19) kennzeichnend für alle Tumoren ist, erklärt werden. Bei Kranken mit Harnblasenkarzinom oder Papillom der Harnblase trifft man oft als Nebenerscheinung eine akute Cystitis an. Die von Terao (2) nachgewiesene erhöhte Adlolaseaktivität bei Patienten mit akuter Cytitis kann, nach unserer Meinung, wohl kaum die Erhöhung der Serumaldolaseaktivität an Kranken mit Harnblasenkarzinom beeinflussen, da die akute Cystitis mit dem Blasenpapillom einhergeht, doch die Werte der Aldolase nahe bei der Norm liegen.

Die Auffassung über die Herkunft der Serumaldolase aus dem Tumorparenchym wird von den Beobachtungen von Sibley und LeHNinger unterstützt, welcher nach operativer Entfernung der Experimentaltumoren an Tieren einc Normalisierung der Serumaldolase feststellen konnten (20).

Die unsererseits beobachteten Veränderungen im Isoenzymmuster der Serumaldolase bei Kranken mit Tumoren im Urogenitalsystem bezichen sich auf die anodisch 
bei der Startlinie liegenden Fraktionen, die vom Typ Muskel „A" Aldolase sind und die nach elektrophoretischer Beweglichkeit den intensivsten Isoenzymfraktionen der Tumoren entsprechen und wahrscheinlich von ihnen abstammen. Diese Veränderungen-sind jedoch nicht spezifisch und können auch bei anderen Erkrankungen, z. B. bösartigen Hämopathien (12) beobachtet werden, was teilweise den diagnostischen Wert der
Untersuchung vermindert. Lediglich an Ratten mit primären, Diäthylnitrosamin-induzierten Leberkarzinomen stellten wir Veränderungen im Isoenzymmuster der Serumaldolase fest, die sich auf die Fraktionen vom Typ Leber Aldolase beziehen, bei keinen anderen Erkrankungen vorkommen und ihren spezifischen Charakters wegen einen hohen diagnostischen Wert besitzen (21).

\section{Literatur}

1. Fedele, L., Acta med. ital., 17, 159 (1962). - 2. Tepao, N., Japan. J. Urol., 59, 91 (1968). - 3. BAKER, R. und D. Govan, Cancer Res., 13, 141 (1953). - 4. BAKER, R., D. Govan, J. HuFfer und J. CAson, J. Clin. Endocr. Springfield, 13, 383 (1953). - 5. Montero, I. und S. D. Perez, Rev. clin. esp., 98, 171 (1965). 6. HahN, M. Cas. lék. česk., 104, 242 (1965). - 7. IANCU, I., C. Feighelis und E. Paúliau, Rev. med.-chir. Nord-Est, 71, 625 (1967). - 8. Krussanowa, N. J. und A. I. Krassowskaja, Vopr. onkol. UdSSR, 9, 9 (1963). - 9. Dikow, A.L., diese Z., 6, 386 (1968). - 10. Dikow, A. L., I. Tschankow und A. SamardjIEw, diese Z., 6, 391 (1968) - 11. Drkow, A. L. und M. Romanow, Zschr. inn. Med., Leipzig, 23, 471 (1968). -12. Dikow, A. L. und
V. Genowa, diese Z. 7, 614 (1969). - 13. Drkow, A. L. und V. Genowa, diese Z., 7, 155 (1969). - 14. TepaO, N., Japan. J.Urol., 59, 98 (1968). - 15. Drkow, A. L., diese Z., 7, 278 (1969). - 16. PANDov, $H$. und A. L. Dikow, Proceedings of the 5th FEBS Meeting, Prag, 1968 (in Druck). - 17. Drkow, A. L., I. LoLova, A. Iwanova und S. Bojrnov, diese Z. 7, 606 (1969). - 18. HadjroLov, D. und A. L. Drkow, Europ. J. Cancer (in Druck). - 19. BoDANSKY, O., Proceedings of the First Conference on Cancer Diagnostic Tests, s. 45, Pub. Health Service, Washington (1950). 20. Siblex, J. A. und A. L. Lehninger, J. Nat. Cancer Inst., Wash., 9, 303 (1949). - 21. Drkow, A. L. und D. Hadjrolov, diese $Z$. $8,38(1970)$.
Dr. med. Angel L. Dikow z. Z. 4630 Bochum-Querenburg Postfach 2148 